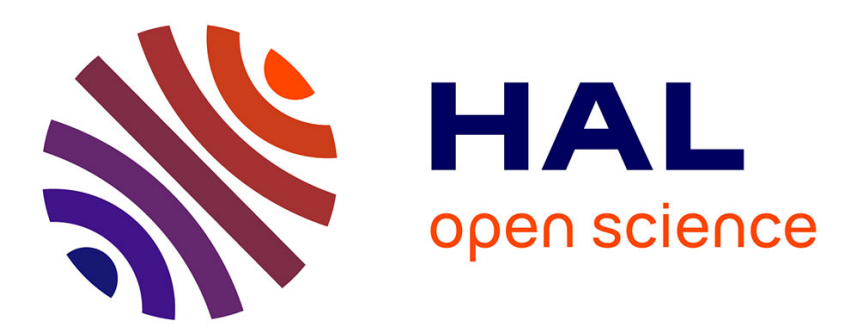

\title{
Lehmann effect in a compensated cholesteric liquid crystal: Experimental evidence with fixed and gliding boundary conditions
}

\author{
Alain Dequidt, Andrzej Zywocinski, Patrick Oswald
}

\section{- To cite this version:}

Alain Dequidt, Andrzej Zywocinski, Patrick Oswald. Lehmann effect in a compensated cholesteric liquid crystal: Experimental evidence with fixed and gliding boundary conditions. European Physical Journal E: Soft matter and biological physics, 2008, 25 (4), pp.277-289. 10.1140/epje/i2007-10290-4 . ensl-00272491

HAL Id: ensl-00272491

https://hal-ens-lyon.archives-ouvertes.fr/ensl-00272491

Submitted on 11 Apr 2008

HAL is a multi-disciplinary open access archive for the deposit and dissemination of scientific research documents, whether they are published or not. The documents may come from teaching and research institutions in France or abroad, or from public or private research centers.
L'archive ouverte pluridisciplinaire HAL, est destinée au dépôt et à la diffusion de documents scientifiques de niveau recherche, publiés ou non, émanant des établissements d'enseignement et de recherche français ou étrangers, des laboratoires publics ou privés. 


\section{EPJ $E$}

\section{Soft Matter}

Eur. Phys. J. E 25, 277-289 (2008)

DOI: $10.1140 /$ epje/i2007-10290-4

Lehmann effect in a compensated cholesteric liquid crystal: Experimental evidence with fixed and gliding boundary conditions

A. Dequidt, A. Żywociński and P. Oswald

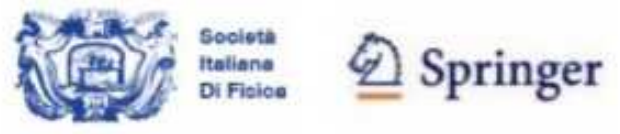




\title{
Lehmann effect in a compensated cholesteric liquid crystal: Experimental evidence with fixed and gliding boundary conditions
}

\author{
A. Dequidt ${ }^{1, a}$, A. Żywociński ${ }^{1,2}$, and P. Oswald ${ }^{1, b}$ \\ 1 Université de Lyon, Laboratoire de Physique, École Normale Supérieure de Lyon, CNRS, 46 Allée d'Italie, 69364 Lyon, France \\ 2 Department III, Institute of Physical Chemistry, Polish Academy of Sciences, Kasprzaka 44/52, 01-224 Warsaw, Poland
}

Received 24 September 2007 and Received in final form 23 November 2007

Published online: 3 April 2008 - (C) EDP Sciences / Società Italiana di Fisica / Springer-Verlag 2008

\begin{abstract}
In a recent letter (Europhys. Lett. 80, 26001 (2007)), we have shown that a compensated cholesteric liquid crystal (in which the macroscopic helix completely unwinds) may be subjected to a thermomechanical torque (the so-called Lehmann effect), in agreement with previous findings of Éber and Jánossy (Mol. Cryst. Liq. Cryst. Lett. 72, 233 (1982)). These results prove that one must take into account the chirality of the molecules and the absence of inversion symmetry at the macroscopic scale when deriving the constitutive equations of the phase at the compensation temperature. In this paper, we present the details of our experimental work and a new experiment performed in a sample treated for planar gliding anchoring. The latter experiment, coupled with a numerical simulation, supports the existence of a thermomechanical coupling in a compensated cholesteric.
\end{abstract}

PACS. 61.30.-v Liquid crystals - 05.70.Ln Nonequilibrium and irreversible thermodynamics - 65.40.De Thermal expansion; thermomechanical effects

\section{Introduction}

When a cholesteric liquid crystal is placed in a temperature gradient parallel to its helical axis, a torque acts on its director $\mathbf{n}$, of expression [1,2]

$$
\boldsymbol{\Gamma}_{\text {Lehmann }}=-\nu \mathbf{G}_{\perp},
$$

where $\mathbf{G}_{\perp}=(\mathbf{n} \times \mathbf{G}) \times \mathbf{n}$ is the component of the temperature gradient $\mathbf{G}=\boldsymbol{\nabla} T$ perpendicular to $\mathbf{n}$. On condition that the director be free to rotate at the boundaries (which is a real problem in experiments), this equation predicts that the helix of a cholesteric liquid crystal must rotate at a constant angular velocity $\Omega$ when it is submitted to a temperature gradient parallel to its axis

$$
\Omega=-\nu G / \gamma_{1},
$$

where $\gamma_{1}$ is the rotational viscosity. Historically, this phenomenon was discovered experimentally by Lehmann at the beginning of the 20th century [3,4] by observing the rotation of the internal texture of cholesteric droplets heated from below and was explained more recently, in 1968, by Leslie [5] from symmetry arguments.

In 1982, Éber and Jánossy proposed to measure the Lehmann coefficient $\nu$ in a compensated cholesteric liquid crystal, i.e. at the special temperature $T_{c}$ at which

\footnotetext{
${ }^{a}$ e-mail: alain.dequidt@ens-lyon.fr

b e-mail: Patrick.Oswald@ens-lyon.fr
}

the equilibrium twist $q=2 \pi / p$ (where $p$ is the cholesteric pitch) vanishes and changes sign $[6,7]$. They found that $\nu$ is different from 0 at $T_{c}$ in spite of the fact that the phase has a nematic-like structure. To prove this result, they performed an ingenious experiment with a cholesteric mixture possessing a compensation temperature, consisting of measuring the birefringence of a homeotropic sample placed in a temperature gradient perpendicular to the director n. Éber and Jánossy found that the director experiences a Lehmann torque $(\nu \neq 0)$ at $T_{c}$ and concluded that it was from microscopic origin and due to the chirality of the molecules. Their experiment was immediately criticized by Pleiner and Brand who claimed that the Lehmann coefficient must vanish at the compensation point $[8,9]$. Their argument (which we will comment in the conclusion) was as follows: "since it is the symmetry of the phase which determines the structure of the macroscopic equations, it is clear that the thermomechanical coupling constant has to vanish at the compensation point, since there the symmetry is exactly that of the nematic phase" [9]. They thus concluded that the result of Éber and Jánossy $(\nu \neq 0)$ was wrong and due to some experimental artifact. This affirmation led to a polemic between theorists and experimentalists, the latter claiming that their results were reliable and not forbidden theoretically because of the chirality of the molecules [10]. 
In view of this controversial situation, we redid the experiment and extended it to another geometry in order to know who was right. Our results were published in a letter [11] and led to the conclusion that molecular chirality was important, in agreement with Éber and Jánossy findings.

On the other hand, our results were reported without details about the experiments and the method for calculating the experimental errors. In this paper we fill this gap and we present a new experiment performed with a sample treated for gliding planar anchoring. This new experiment together with a numerical simulation have confirmed our previous conclusions.

The plan of the article is the following. In Section 2, we recall the principle of the Éber and Jánossy experiment in homeotropic anchoring and we show that it can be extended to the planar geometry. We then calculate for each geometry the director distortions in the temperature gradient and the expression for the phase shift between the ordinary and extraordinary components of a laser beam crossing the sample at the compensation temperature. We show that this shift depends on the optical indices, on the elastic constants and on an effective Lehmann coefficient of expression $\nu_{\text {eff }}=\nu+K_{2} \mathrm{~d} q / \mathrm{d} T$, where $K_{2}$ is the twist Frank constant. In Section 3, we describe the experiment and we deduce from the measurement of the optical phase shifts two different combinations of the effective Lehmann coefficient with the optical indices and the two other Frank constants $K_{1}$ and $K_{3}$. In Section 4, we explain how we measured $\mathrm{d} q / \mathrm{d} T$ as well as the elastic constants $K_{i}(i=1,2,3)$ and the optical indices. In Section 5 , we give the value of the Lehmann coefficient $\nu$ and we recall the principle of the maximum-likelihood method used to calculate the error bars. In Section 6 a new experiment performed with a sample treated for gliding planar anchoring is described. After characterizing the anchoring, we show the existence of a continuous rotation of the director at the compensation point when the sample is placed in a vertical temperature gradient. A simple model and a numerical simulation allow us to reproduce the observations qualitatively. Finally, we draw conclusions about the role of the molecular chirality in Section 7 .

\section{The Éber and Jánossy experiment: theory}

In the original experiment of Éber and Jánossy, the cholesteric liquid crystal is introduced between two parallel glass plates treated for strong homeotropic anchoring. The sample is then placed inside a temperature gradient parallel to the glass plates. In this geometry, the cholesteric helix unwinds when its pitch is typically larger than the sample thickness. As a consequence a band of homeotropic nematic phase, centered on the compensation temperature, forms in the sample. This region is bordered by cholesteric fingers which are well visible under the microscope (for a review about the helix unwinding, see $[2,12]$ ) (Fig. 1). In practice, the nematic phase is a little distorted because of the presence of the temperature

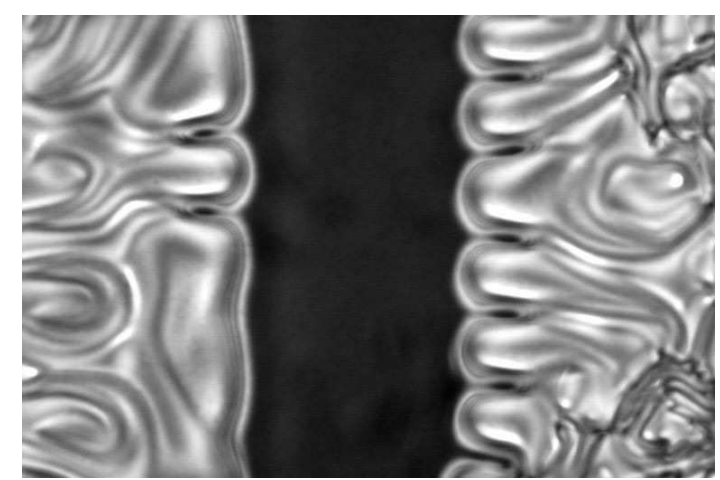

Fig. 1. Homeotropic sample in a large temperature gradient $\left(d=40 \mu \mathrm{m}\right.$ and $\left.G=51{ }^{\circ} \mathrm{C} / \mathrm{cm}\right)$. The unwound zone is $465 \mu \mathrm{m}$ wide.

gradient. The director field distortions are obtained by solving the torque equilibrium equations. We recall that in our notations, $\mathbf{n} \cdot \nabla \times \mathbf{n}=-q$ at equilibrium. To first order in temperature gradient $G$, they read

$$
\begin{gathered}
2 K_{2} q \frac{\partial n_{y}(x, z)}{\partial z}+K_{3} \frac{\partial^{2} n_{x}(x, z)}{\partial z^{2}}+K_{1} \frac{\partial^{2} n_{x}(x, z)}{\partial x^{2}}=0 \\
G \nu_{e f f}+K_{3} \frac{\partial^{2} n_{y}(x, z)}{\partial z^{2}} \\
+K_{2}\left(\frac{\partial^{2} n_{y}(x, z)}{\partial x^{2}}-2 q \frac{\partial n_{x}(x, z)}{\partial z}\right)=0
\end{gathered}
$$

with $\nu_{\text {eff }}=\nu+\frac{\mathrm{d} K_{2} q}{\mathrm{~d} T}$. The $x$-axis is chosen parallel to the temperature gradient and the $z$-axis perpendicular to the glass plates. The system is invariant along the $y$-direction.

In practice, second derivatives with respect to $x$ can be neglected, which can be justified a posteriori (the sample thickness $d$ is always much smaller than the width of the nematic band) so that the previous equations become:

$$
\begin{aligned}
& 2 K_{2} q \frac{\partial n_{y}(x, z)}{\partial z}+K_{3} \frac{\partial^{2} n_{x}(x, z)}{\partial z^{2}}=0 \\
& G \nu_{e f f}+K_{3} \frac{\partial^{2} n_{y}(x, z)}{\partial z^{2}}-2 q K_{2} \frac{\partial n_{x}(x, z)}{\partial z}=0 .
\end{aligned}
$$

Solving these equations gives

$$
\begin{aligned}
& n_{x}=\frac{G \nu_{e f f}}{2 K_{2} q} d\left(\frac{z}{d}-\frac{1}{2}+\frac{1}{2} \frac{\sin \left(q(d-2 z) \frac{K_{2}}{K_{3}}\right)}{\sin \left(q d \frac{K_{2}}{K_{3}}\right)}\right), \\
& n_{y}=\frac{G \nu_{e f f}}{2 K_{2} q} d \frac{\sin \left(q z \frac{K_{2}}{K_{3}}\right) \sin \left(q(d-z) \frac{K_{2}}{K_{3}}\right)}{\sin \left(q d \frac{K_{2}}{K_{3}}\right)} .
\end{aligned}
$$

These equations generalize the solution given by Éber and Jánossy [6] since they are still valid out of the compensation point $T_{c}$. In particular, they give back the spinodal limit for the nematic phase as $n_{x}$ and $n_{y}$ diverge when $q d=\pi\left(K_{3} / K_{2}\right)$ or $d / p=K_{3} /\left(2 K_{2}\right)$.

The solution can be linearized in $q$ in the vicinity of the compensation temperature (at which $q=0$ ), which 
gives

$n_{x}=\frac{G \nu_{e f f} K_{2}}{3 K_{3}^{2}} q z\left(z-\frac{d}{2}\right)(z-d), \quad n_{y}=\frac{G \nu_{e f f}}{2 K_{3}} z(d-z)$.

This distortion of the director field can be detected optically by measuring the phase shift $\Phi_{\mathrm{H}}$ between the ordinary and the extraordinary components of a laser beam crossing the sample. In practice, the beam is never strictly perpendicular to the sample, which may be a source of error. For this reason, we calculated the phase shift at the compensation temperature by taking into account a small misalignment of the laser beam. Let $\theta$ be the angle (assumed to be small) between the beam and the normal to the sample and $\varphi$ the azimuthal angle of the beam with respect to the temperature gradient. A straightforward calculation yields:

$$
\begin{aligned}
\Phi_{\mathrm{H}}= & -\left(\frac{G \nu_{e f f}}{K_{3}} d^{2}\right)^{2} \frac{n_{e}^{2}-n_{o}^{2}}{240 n_{e}^{2}} k n_{o} d \\
& +\frac{G \nu_{e f f}}{K_{3}} d^{2} \frac{n_{e}^{2}-n_{o}^{2}}{12 n_{e}^{2}} \theta(\sin \varphi) k d,
\end{aligned}
$$

where $n_{o}$ and $n_{e}$ are the ordinary and extraordinary indices, respectively, $k=2 \pi / \lambda$ the angular wave number of the laser, while $\nu_{e f f}=\nu+K_{2} \frac{\mathrm{d} q}{\mathrm{~d} T}$ at $T_{c}$ as $q=0$. This equation shows that, at normal incidence $(\theta=0), \Phi_{\mathrm{H}}$ is proportional to $G^{2}$ and $d^{5}$, a result already given by Éber and Jánossy in reference [6]. On the other hand, an additional term linear in $G$ appears when the laser beam is slightly misaligned, but the term in $G^{2}$ remains unchanged.

This experiment can be also performed in planar anchoring, provided that the molecular alignment direction be perpendicular to the temperature gradient. In this geometry, a nematic band forms which is centered on the inversion temperature. At equilibrium, this band is bordered by two $\chi$-disclination lines behind which regions twisted by $2 \pi$ form (Fig. 2).

As in the homeotropic case, the nematic is distorted by the temperature gradient. The governing equations for the director field become in this case

$$
\begin{gathered}
K_{2}\left(\frac{\partial^{2} n_{x}}{\partial z^{2}}-\frac{\partial^{2} n_{z}}{\partial z \partial x}\right)+K_{1}\left(\frac{\partial^{2} n_{x}}{\partial x^{2}}+\frac{\partial^{2} n_{z}}{\partial z \partial x}\right)=0 \\
-G \nu_{e f f}+K_{1}\left(\frac{\partial^{2} n_{z}}{\partial z^{2}}+\frac{\partial^{2} n_{x}}{\partial x \partial z}\right) \\
+K_{2}\left(\frac{\partial^{2} n_{z}}{\partial x^{2}}-\frac{\partial^{2} n_{x}}{\partial x \partial z}\right)=0
\end{gathered}
$$

with, as before, $\nu_{\text {eff }}=\nu+\frac{\mathrm{d} K_{2} q}{\mathrm{~d} T}$. In practice $\partial / \partial x \ll$ $\partial / \partial z$, which allows us to simplify the previous equations as follows:

$$
\begin{aligned}
& K_{2} \frac{\partial^{2} n_{x}}{\partial z^{2}}=0 \\
& -G \nu_{e f f}+K_{1} \frac{\partial^{2} n_{z}}{\partial z^{2}}=0
\end{aligned}
$$

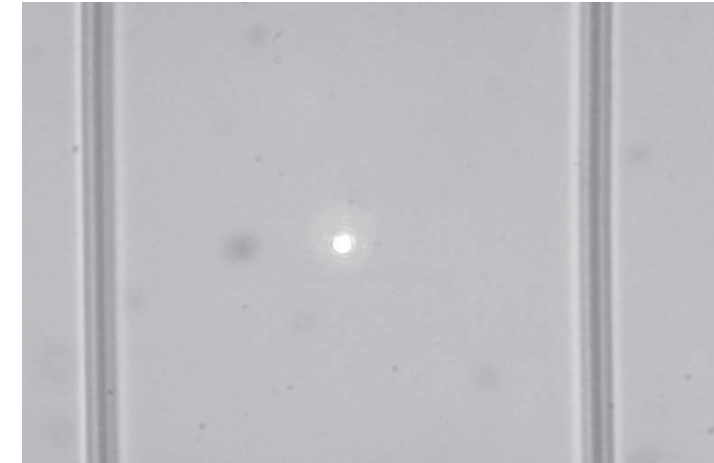

Fig. 2. Planar sample in a moderate temperature gradient $\left(d=58 \mu \mathrm{m}\right.$ and $\left.G=7.2^{\circ} \mathrm{C} / \mathrm{cm}\right)$. The anchoring direction is vertical and the temperature gradient horizontal. The unwound zone between the two $\chi$-disclination lines is $1100 \mu \mathrm{m}$ wide. In the center, one can see the image of the laser beam (highly attenuated to not saturate the camera).

The solution satisfying boundary conditions reads simply

$$
n_{x}=0 \quad \text { and } \quad n_{z}=-\frac{G \nu_{e f f}}{2 K_{1}} z(d-z)
$$

These distortions can be detected by measuring the phase shift $\Phi_{\mathrm{P}}$ between the ordinary and extraordinary components of a laser beam crossing the sample. A straightforward calculation gives in this case at the compensation temperature:

$$
\begin{aligned}
\Phi_{\mathrm{P}}= & -\psi+\left(\frac{G \nu_{e f f}}{K_{1}}\right)^{2} \frac{n_{e}^{2}-n_{o}^{2}}{240 n_{o}^{2}} k n_{e} d^{5}-\frac{G \nu_{e f f}}{K_{1}} \theta \\
& \times\left[\frac{n_{e}^{2}-n_{o}^{2}}{12 n_{o}^{2}} k d^{3} \sin \varphi\right. \\
& \left.+\left(\frac{d \cos ^{2} \frac{\psi}{2}}{k n_{o}^{2}}-\frac{\sin \psi}{k^{2} n_{o}^{2}\left(n_{e}-n_{o}\right)}\right) \cos \varphi\right],
\end{aligned}
$$

with $\psi=k d\left(n_{e}-n_{o}\right)$. As in the homeotropic case, the phase shift contains (in addition to the constant term $-\psi$ ) a linear term in $G$ which vanishes when $\theta=0$ and a quadratic term in $G$ proportional to $d^{5}$ and independent of angles $\theta$ and $\varphi$.

From these calculations, we see that measuring $\Phi_{\mathrm{H}}$ and $\Phi_{\mathrm{P}}$ on the one hand, and $n_{o}, n_{e}, K_{i}(i=1,2,3)$ and $\mathrm{d} q / \mathrm{d} T$ on the other hand, allows us to determine the Lehmann coefficient $\nu$. In the next section, we present our experiment and recall how to measure $\Phi_{\mathrm{H}}$ and $\Phi_{\mathrm{P}}$.

\section{Experiment}

Our liquid crystal is a mixture of 4- $n$-octyloxy- $4^{\prime}$ cyanobiphenyl (8OCB from Synthon Chemicals GmbH \& Co) and of cholesteryl chloride (CC from Aldrich) in proportion 1:1 in weight with a compensation point at $59^{\circ} \mathrm{C}$ and a clearing point at $67^{\circ} \mathrm{C}$. The $8 \mathrm{OCB}$ was purified by one of us (AZ) and the CC was used without further purification. For practical reasons, we did not use the mixture 
of Éber and Jánossy, namely 8CB + CC in equal weight proportions, because the $\mathrm{CC}$ crystallizes very rapidly in this mixture at the compensation temperature (close to $40^{\circ} \mathrm{C}$ ). This problem was considerably reduced by replacing $8 \mathrm{CB}$ by $8 \mathrm{OCB}$, certainly because the compensation temperature was higher by about $20^{\circ} \mathrm{C}$. It was nonetheless crucial (to avoid the crystallization of the CC) to melt and systematically homogenize the mixture at high temperature (typically during $5 \mathrm{~min}$ at $100^{\circ} \mathrm{C}$ ) before filling each sample by capillarity at about $60^{\circ} \mathrm{C}$. We also took care to prepare the mixture in small quantities to limit its degradation, pretty fast when it is heated above $100^{\circ} \mathrm{C}$. The mixture was also filtered in the cholesteric phase through $0.2 \mu \mathrm{m}$ nylon filter to remove the maximum number of dust particles. The glass plates were treated either for homeotropic anchoring with DMOAP (according to the Kahn procedure [13]) or for planar anchoring with a rubbed polyimide layer baked at $300^{\circ} \mathrm{C}$ during 2 hours (ZLI 2650 from Merck). Nickel wires of calibrated diameters were used as spacers to fix the sample thickness. The temperature gradient was imposed by placing the sample in a directional solidification cell (for its description, see Ref. [14]), itself mounted on the stage of a polarizing microscope. A semi-reflecting plate was placed under the condensor of the microscope to illuminate the sample with a He-Ne laser $(2 \mathrm{~mW}, \lambda=633 \mathrm{~nm})$. The laser beam was first expanded to $6 \mathrm{~mm}$ with a commercial beam expander and then it was focused inside the sample with the condensor. Its diameter at the waist was of the order of $5 \mu \mathrm{m}$ and did not change significantly over the sample thickness (ranging between 50 and $110 \mu \mathrm{m}$ ). An $x-y$ translation stage allowed manual positioning of the cell in order that the laser spot lied exactly in the middle of the nematic band, at the compensation temperature $T_{c}$. The phase shift between the ordinary and extraordinary components of the laser beam crossing the sample was measured using a rotating analyzer, a quarter-wave plate, a photodiode and a lock-in amplifier following the method of Lim and Ho [15]. A synoptic of experimental setup is shown in Figure 3.

\subsection{Results in homeotropic anchoring}

An example of curve obtained with a homeotropic $100 \mu \mathrm{m}-$ thick sample is shown in Figure 4 . The solid line represents the best fit of the data to a parabola $\Phi_{\mathrm{H}}=a_{\mathrm{H}} G-b_{\mathrm{H}} G^{2}$. According to equation (10), $a_{\mathrm{H}}=\frac{\nu_{e f f}}{K_{3}} \frac{n_{e}^{2}-n_{o}^{2}}{12 n_{e}^{2}} \theta \sin \varphi k d^{3}$ and $b_{\mathrm{H}}=\left(\frac{\nu_{e f f}}{K_{3}}\right)^{2} \frac{n_{e}^{2}-n_{o}^{2}}{240 n_{e}^{2}} k n_{o} d^{5}$. In Figure 5, we plotted the fit parameter $b_{\mathrm{H}}$ as a function of the sample thickness $d$. Within experimental errors, $b_{\mathrm{H}}$ is proportional to $d^{5}$ as predicted by the theory [16]. Fitting $b_{\mathrm{H}}$ with a linear law in $d^{5}$ leads to

$$
\left(\frac{\nu_{e f f}}{K_{3}}\right)^{2} \frac{n_{e}^{2}-n_{o}^{2}}{240 n_{e}^{2}} k n_{o}=(9,3 \pm 0.8) \times 10^{13} \mathrm{rad} \mathrm{K}^{-2} \mathrm{~m}^{-3} .
$$

As for values of $a_{\mathrm{H}}$ obtained from the fits, they give expected typical angles $\theta \approx 1-3^{\circ}$.

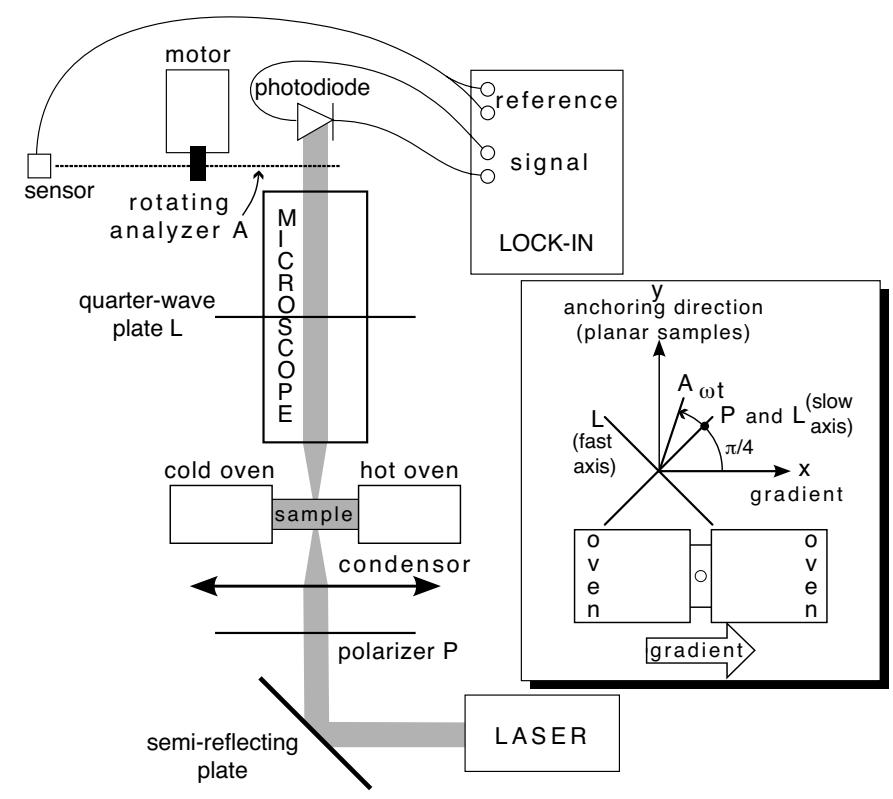

Fig. 3. Schematic diagram of the experimental setup used to measure the phase shift between the ordinary and extraordinary components of the laser beam crossing the sample. In practice, we chose $\omega / 2 \pi \approx 100 \mathrm{~s}^{-1}$.

We also studied the influence of a shift in temperature with respect to $T_{c}$ on the value of $\Phi_{\mathrm{H}}$. We checked that the signal does not change in a measurable way when the laser spot is slightly decentred with respect to $T_{c}$ (by no more than $\pm 0.05^{\circ} \mathrm{C}$ ). On the other hand, $\Phi_{\mathrm{H}}$ starts to decrease for larger shift in a significant way, in particular when approaching the fingers. In the same time the signal becomes noisy because it starts to depend on the position of the spot along the $y$-direction (perpendicular to the temperature gradient). We nevertheless checked that in a systematic way, $\Phi_{\mathrm{H}}$ passes through a maximum at middistance of the fingers (i.e., at temperature $T_{c}$ ). A typical curve is shown in Figure 6. It is well fitted by a parabola of type $\Phi_{\mathrm{H}}=\Phi_{\mathrm{H}}\left(T_{c}\right)+a\left(T-T_{c}\right)^{2}$.

In order to exploit this experimental result, we looked theoretically for the temperature dependence of $\Phi_{\mathrm{H}}$ by taking into account the local tilt of the director given by equations (7) and (8). In principle, the phase shift may be expanded in a power series of $q$ around the compensation point as follows:

$$
\Phi_{\mathrm{H}}\left(q, \nu_{e f f}\right)=a\left(\nu_{e f f}\right)+b\left(\nu_{e f f}\right) q+c\left(\nu_{e f f}\right) q^{2}+\ldots
$$

where $a\left(\nu_{e f f}\right)=\Phi_{\mathrm{H}}\left(0, \nu_{\text {eff }}\right)$ is given by equation (10). This term, as well as $b$ and $c$, are functions of $d, G$ and the material constants $K_{1}, K_{2}, K_{3}, n_{o}$ and $n_{e}$ which we assume to be independent of the temperature in the vicinity of $T_{c}$. A straightforward calculation at first order in $q$ showed that $b$ is strictly null. As a consequence, $\Phi_{\mathrm{H}}$ must pass through a maximum at a temperature $T_{\max }$ solution 


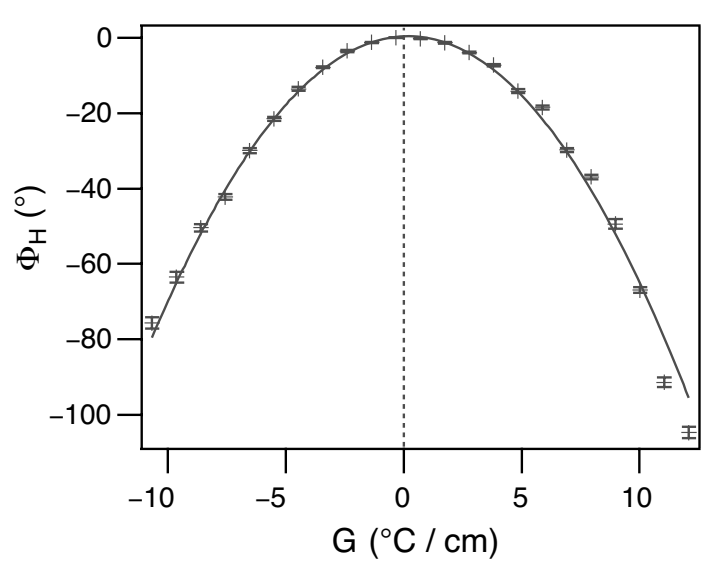

Fig. 4. Phase shift as a function of the temperature gradient ( $d=100 \mu \mathrm{m}$, homeotropic anchoring).

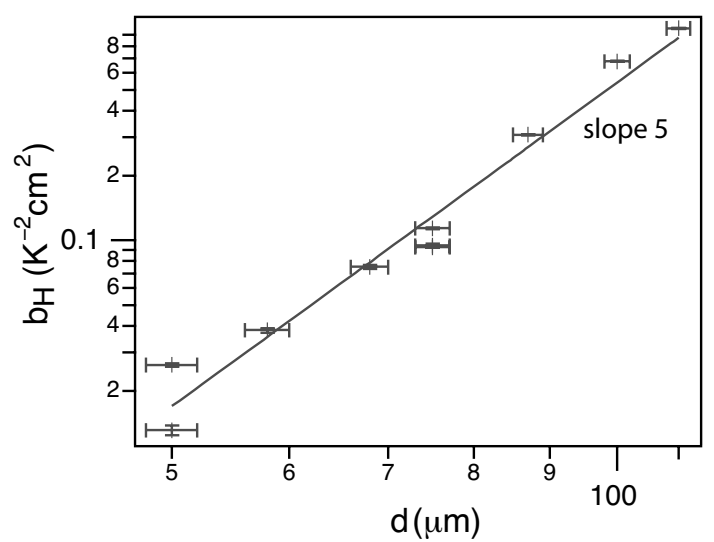

Fig. 5. Fit parameter $b_{\mathrm{H}}$ as a function of the sample thickness $d$. The $d^{5}$-dependence is well satisfied.

of equation

$$
\frac{\partial \Phi_{\mathrm{H}}}{\partial T}=\frac{\partial a}{\partial \nu_{e f f}} \frac{\partial \nu_{e f f}}{\partial T}+\frac{\partial c}{\partial \nu_{e f f}} \frac{\partial \nu_{e f f}}{\partial T} q^{2}+2 c q \frac{\partial q}{\partial T}=0
$$

Experimentally, $T_{\max }=T_{c}$. As $q=0$ at this temperature, we deduce from the previous equation that

$$
\frac{\partial \nu_{e f f}}{\partial T}=0
$$

Because $\mathrm{d} q / \mathrm{d} T \neq 0$ at $T_{c}$, this implies that

$$
\frac{\partial \nu_{e f f}}{\partial q}=0
$$

Consequently, we find that $\nu_{\text {eff }}$ is independent of $q$ within our experimental errors. On the other hand, we cannot conclude at this stage that the Lehmann coefficient $\nu$ is null at $T_{c}$. This point will be addressed later, once the corrective term $K_{2} \frac{\mathrm{d} q}{\mathrm{~d} T}$ has been measured.

\subsection{Results in planar anchoring}

Experiments in planar geometry were much more delicate to perform than the previous ones for several reasons.

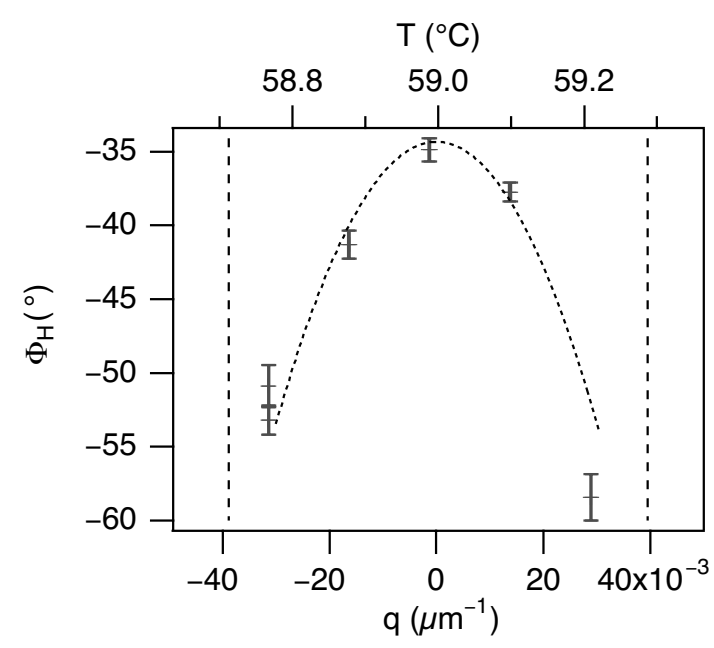

Fig. 6. Phase shift $\Phi_{\mathrm{H}}$ as a function of temperature $T$ and equilibrium twist $q$. The data fit reasonably with a parabola with the maximum at $T_{c}$ within experimental errors. The dashed vertical lines mark the limits between the nematic band and the fingers. The sample thickness is $d=87 \mu \mathrm{m}$ and the temperature gradient $G=11^{\circ} \mathrm{C} / \mathrm{cm}$.

The first one came from the difficulty to prepare thick planar samples in the "nematic" phase at the compensation temperature. For this purpose, we used a Mettler oven in which the sample can be moved easily in order to visualize its texture under the microscope. In practice, we did not succeed to prepare samples thicker than $70 \mu \mathrm{m}$. The reason was mainly due to the difficulty for the $\chi$ disclination lines to move in thick samples because they trap dust particles, which finally stop their motion. Another difficulty was to be sure that the sample, once prepared, was well in the "nematic" phase. Indeed, a thick sample twisted by $\pm \pi$ within the thickness has the same optical contrast between crossed polarizer and analyzer as a "nematic" sample because of the adiabatic rotation of the plane of polarization of the light. To know the director orientation, we used the following method. The sample was rapidly heated until destabilization occurs: during this process, bands develop, which are perpendicular to the director orientation in the middle of the sample. It was then enough to look at the band orientation as bands perpendicular to the anchoring direction indicates that the sample is well in the nematic phase, whereas bands parallel to the anchoring direction reveals a $\pm \pi$-twist of the director field. Once the "nematic" sample was prepared, it was transfered as fast as possible into the directional solidification cell. Because of the temperature gradient, the sample immediately destabilized on both sides of the "nematic" central zone. This led first to periodic bands parallel to the temperature gradient which progressively disappeared by leaving two zones twisted respectively by $2 \pi$ and $-2 \pi$. After a transient of a few hours, all the stripes disappeared, leaving a "nematic" zone separated from two twisted zones by two non singular double $\chi$-disclination lines [2]. These two lines are well visible in Figure 2. 


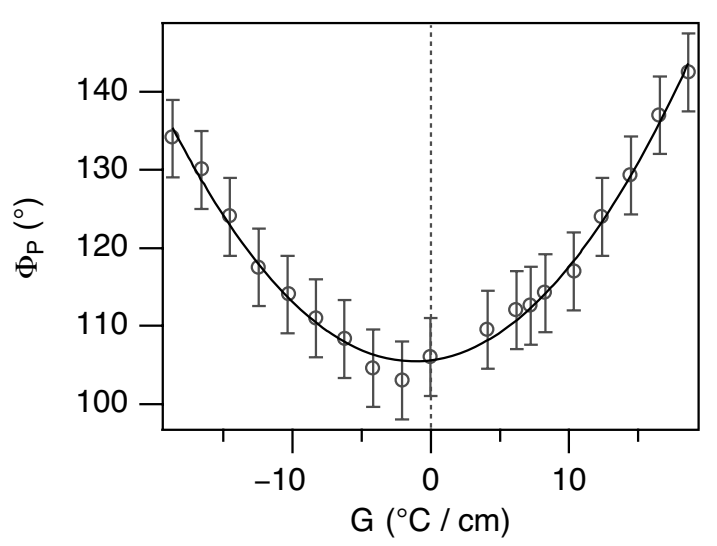

Fig. 7. Phase shift as a function of the temperature gradient (d=58 $\mu \mathrm{m}$, planar anchoring).

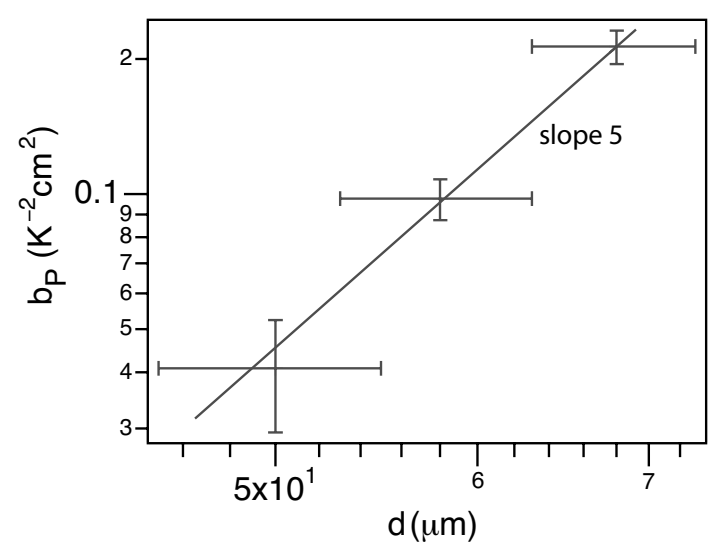

Fig. 8. Fit parameter $b_{\mathrm{P}}$ as a function of the sample thickness $d$. The $d^{5}$-dependence is still well satisfied.

The second problem was due to the presence of a constant term in the phase shift $\Phi_{\mathrm{P}}$ measured experimentally. Indeed, the term $-\psi=-k d\left(n_{e}-n_{o}\right)$ appears in the expression of $\Phi_{\mathrm{P}}$ as we can see in equation (16). This term is very large (of the order of $20 \pi$ ) in comparison with the variations we want to measure, less than $1 \mathrm{rad}$. In order to avoid that this term changes, we took care to perform all the measurements at the same place in the sample (so that $d$ is constant). It was also crucial before each measurement to check that the laser spot was exactly at equal distances of the two disclination lines bordering the "nematic" zone. This condition was achieved by finely adjusting the two oven temperatures and was essential to avoid that the birefringence changes. Finally, it was important to perform measurements as fast as possible to avoid any variation in the birefringence due to a degradation of the sample. With these precautions, it was possible to consider that the term $-\psi$ in $\Phi_{\mathrm{P}}$ was constant.

An example of curve obtained with a planar $58 \mu \mathrm{m}$ thick sample is shown in Figure 7 . The solid line represents the best fit of the data to a parabola $\Phi_{\mathrm{P}}=$ $a_{\mathrm{P}} G-b_{\mathrm{P}} G^{2}+$ c. According to equation (16), $a_{\mathrm{P}}=$ $-\frac{\nu_{e f f}}{K_{1}} \theta\left[\frac{n_{e}^{2}-n_{o}^{2}}{12 n_{o}^{2}} k d^{3} \sin \varphi+\left(\frac{d \cos ^{2} \frac{\psi}{2}}{k n_{o}^{2}}-\frac{\sin \psi}{k^{2} n_{o}^{2}\left(n_{e}-n_{o}\right)}\right) \cos \varphi\right]$, $b_{\mathrm{P}}=\left(\frac{\nu_{e f f}}{K_{1}}\right)^{2} \frac{n_{e}^{2}-n_{o}^{2}}{240 n_{o}^{2}} k n_{e} d^{5}$ and $c=-\psi$.

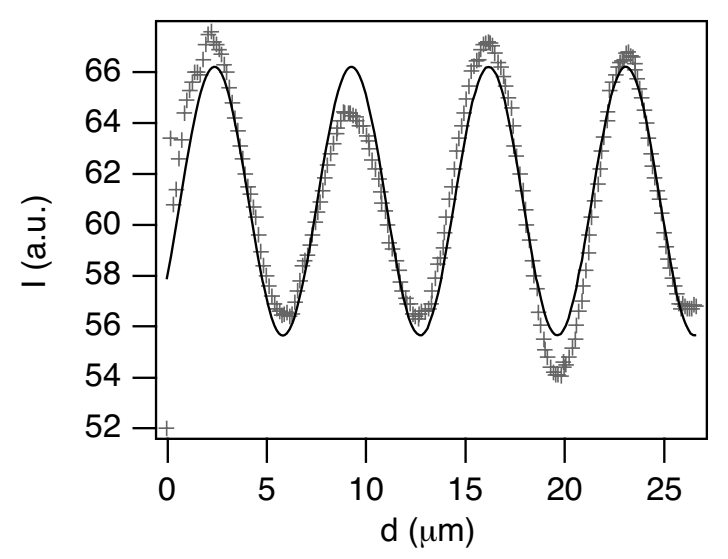

Fig. 9. Transmitted intensity between crossed polarizers as a function of the local thickness in a wedge planar sample.

In Figure 8, we plotted the fit parameter $b_{\mathrm{P}}$ as a function of the sample thickness $d$. Within experimental errors, $b_{\mathrm{P}}$ is proportional to $d^{5}$ as predicted by the theory [17]. Fitting $b_{\mathrm{P}}$ with a linear law in $d^{5}$ leads to

$$
\left(\frac{\nu_{e f f}}{K_{1}}\right)^{2} \frac{n_{e}^{2}-n_{o}^{2}}{240 n_{o}^{2}} k n_{e}=(25 \pm 6) \times 10^{13} \mathrm{rad} \mathrm{K}^{-2} \mathrm{~m}^{-3}
$$

The next step was to measure the material constants $n_{o}, n_{e}, K_{1}, K_{2}, K_{3}$ and $\mathrm{d} q / \mathrm{d} T$ at the compensation temperature. Our results are given in the next section.

\section{Material constants}

Great care was taken to measure these coefficients. Details of our experiments are given below.

The birefringence $\Delta n=n_{e}-n_{o}$ was obtained by measuring with a CCD camera the transmitted intensity between crossed polarizers of a He-Ne laser as a function of the local thickness in a wedge planar sample $(0$ to $30 \mu \mathrm{m})$. The thickness profile of the empty cell was previously measured in green light $(546 \mathrm{~nm})$ by determining the positions of the equal-thickness fringes with a reflecting Leica macroscope. Our measurements are reported in Figure 9. The best fit to a law of type $I=a+b \cos (c d+f)$ (with $c=2 \pi \Delta n / \lambda$ and $\lambda=632.8 \mathrm{~nm}$ ) gives

$$
\Delta n=0,0917 \pm 0.0003
$$

The indices were then deduced separately by measuring the phase shift $\psi$ between the ordinary and extraordinary components of a He-Ne laser beam crossing homeotropic samples of different thicknesses at an incidence angle $i=15^{\circ} 10^{\prime}$. We recall that $\psi$ has for expression $\psi=k n_{o} d\left(\sqrt{1-\frac{\sin ^{2} i}{n_{e}^{2}}}-\sqrt{1-\frac{\sin ^{2} i}{n_{o}^{2}}}\right)$. Our data " $\psi$ as a function of $d "$ are shown in Figure 10. From their fit to a linear law and the previous value of the birefringence, we obtain

$$
n_{o}=1.55 \pm 0.01 \quad \text { and } \quad n_{e}=1.64 \pm 0.01 \text {. }
$$




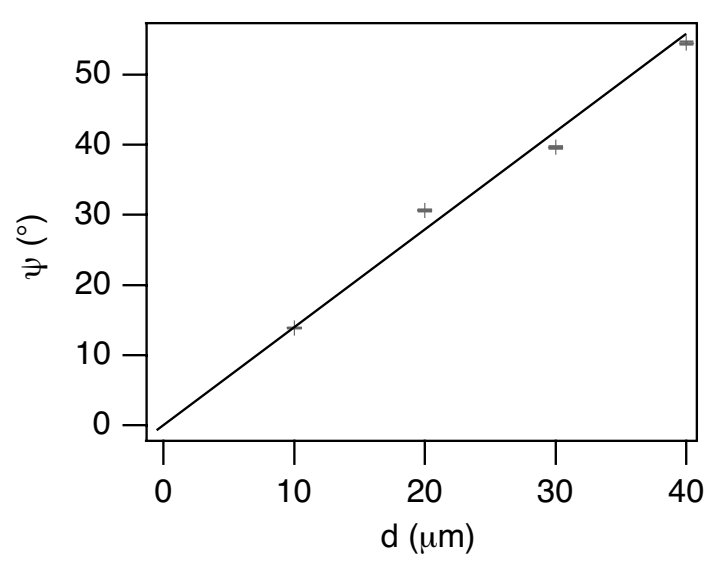

Fig. 10. Phase shift between the ordinary and extraordinary rays as a function of the sample thickness at oblique incidence (homeotropic anchoring).

The pitch was measured as a function of temperature by using commercial cells (from Instec, Inc) treated for planar anchoring. The cells contain silica balls which allow to fix their thickness accurately $(5,6.8,9$ or $20 \pm 0.2 \mu \mathrm{m})$. After filling, the cells were placed in a precision homemade oven both regulated and homogenous in temperature within $0.01{ }^{\circ} \mathrm{C}$. The absolute value of the pitch was measured by looking for the particular temperatures at which two zones with two different values of the twist coexist. Let $n$ and $n^{\prime}$ be the numbers of half pitches in two adjacent zones. It can be easily checked that the two zones have exactly the same elastic energy at temperature $T$ such that $q(T)=\frac{\left(n+n^{\prime}\right) \pi}{2 d}$. In general, $\left|n-n^{\prime}\right|=1$ (sometimes 2). Figure 11 shows an example of a $20 \mu \mathrm{m}-$ thick sample in which an unwound zone (nematic phase, black between crossed polarizers) is in equilibrium with a half-pitch twisted zone. In this example, the temperature is adjusted in order that the $\chi$-line separating the two zones form a broken line joining the silica balls. By contrast, the line segments bend visibly in the direction of the zone of lower energy when the temperature is changed by typically $\pm 0.02{ }^{\circ} \mathrm{C}$. This method allowed us to measure $q(T)$ (Fig. 12). The best fit of the experimental data to a polynomial of degree $2: q=a\left(T-T_{c}\right)+b\left(T-T_{c}\right)^{2}$ led to $a=\frac{\mathrm{d} q}{\mathrm{~d} T}\left(T=T_{c}\right)=0.1365 \pm 0.001 \mu \mathrm{m}^{-1} \mathrm{~K}^{-1}$. The sign of $q$ was determined using a cell with a strong planar anchoring at the bottom plate and a gliding planar anchoring at the top plate.

In order to measure the elastic constants, we used three different methods.

First, we measured the Fréedériks transition in planar samples of different thicknesses $(6.8,10$, and $20 \mu \mathrm{m})$ using a capacitive method (see Fig. 13). From these experiments (and capacitance measurements of homeotropic samples) we deduced successively the dielectric constants

$$
\varepsilon_{\|}=9.4 \pm 0.5 \quad \text { and } \quad \varepsilon_{\perp}=4.5 \pm 0.2,
$$

the splay constant, or equivalently

$$
\frac{K_{1}}{\varepsilon_{0} \varepsilon_{a}}=0.076 \pm 0.006 \mathrm{~V}^{2}
$$
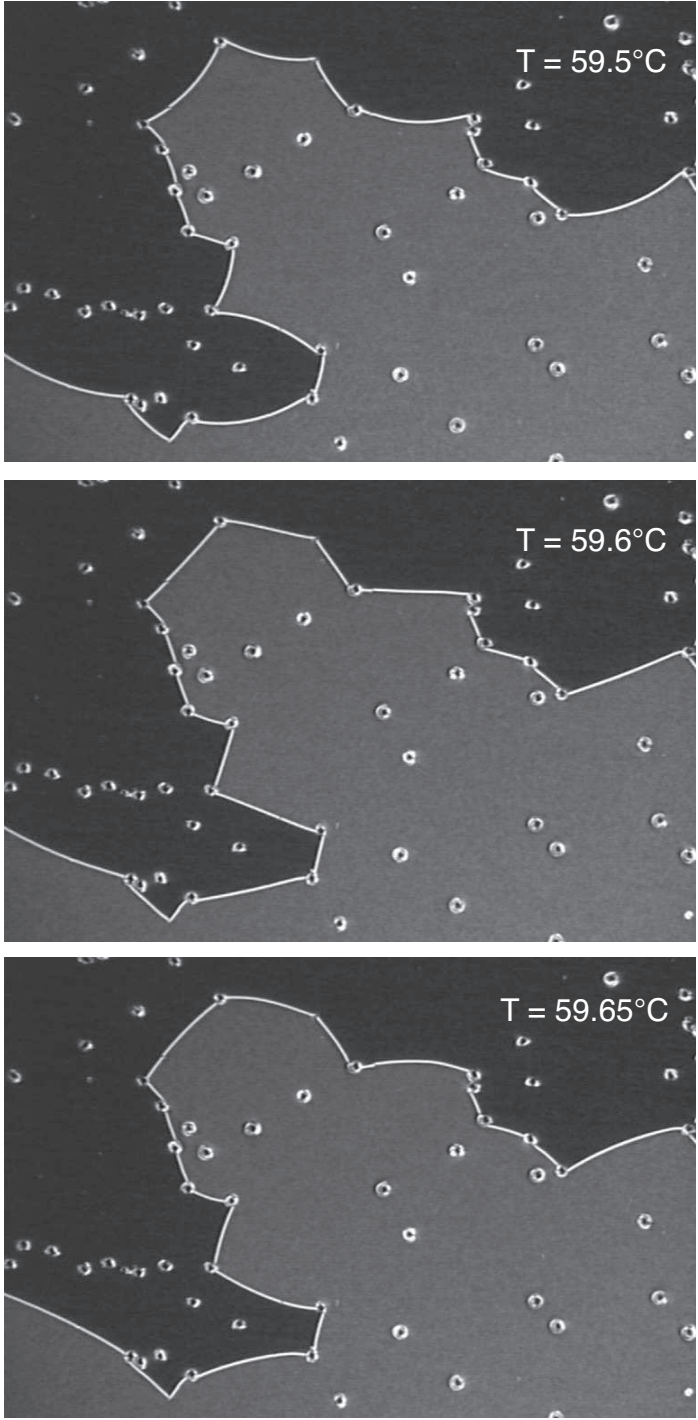

Fig. 11. Three photographs taken at slightly different temperatures of the broken line separating an unwound zone (nematic phase, black) from a half-pitch twisted zone (grey). The line is pinned on the balls used to fix the sample thickness. At $T=59.6{ }^{\circ} \mathrm{C}$, the line segments are straight, indicating that the two zones have exactly the same energy $(d=20 \mu \mathrm{m})$.

with $\varepsilon_{a}=\varepsilon_{\|}-\varepsilon_{\perp}$, as well as the ratio of the bend and splay constants

$$
\frac{K_{3}}{K_{1}}=1.7 \pm 0.1
$$

Note that $K_{1}$ was obtained from the direct measurement of the critical voltage (given by $V_{c}=\pi \sqrt{\frac{K_{1}}{\varepsilon_{0} \varepsilon_{a}}}$ ), while the ratio $K_{3} / K_{1}$ was deduced from the fit of the whole capacitance- $v s$.-voltage curve according to the procedure given in [18]. We emphasize that we neglected the flexoelectric effects in the fitting procedure, which is justified as long as the flexoelectric coefficient difference $e_{3}-e_{1}$ is smaller than $10^{-11} \mathrm{Cm}^{-1}[19]$. This is the case in our mixture, in which we measured $e_{3}-e_{1}=3 \times 10^{-12} \mathrm{Cm}^{-1}[20]$. 


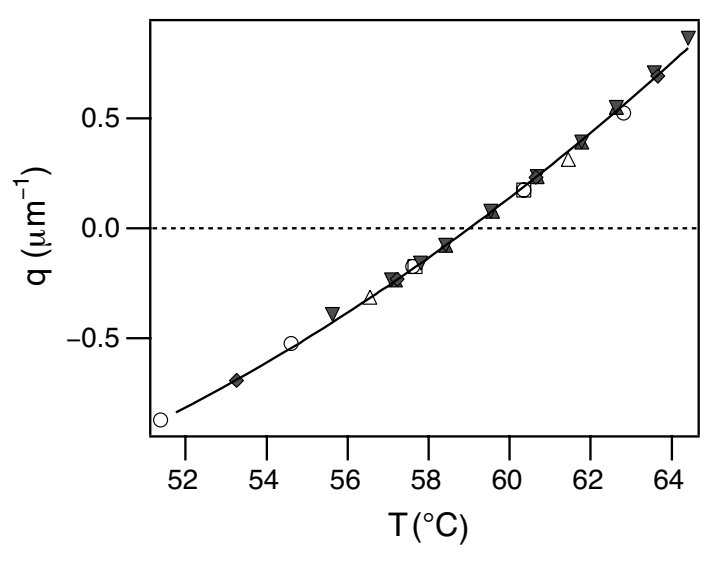

Fig. 12. Equilibrium twist as a function of temperature. The different symbols correspond to samples of different thicknesses $(5,6.8,9$ and $20 \mu \mathrm{m})$.

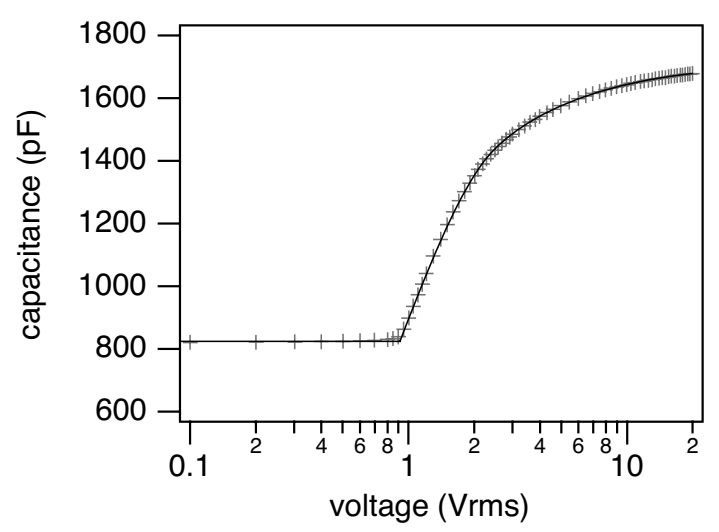

Fig. 13. Cell capacitance as a function of the applied voltage $(d=10 \mu \mathrm{m}, f=10 \mathrm{kHz})$. The solid line is the best fit to the theory.

Second, we measured the Fréedériks transition in a $\pi / 2$-twisted planar cell (thickness $6.8 \mu \mathrm{m}$, from Instec, Inc) at different temperatures close to the compensation point. Curves are shown in Figure 14. In this geometry, the critical voltage is given by [21]

$$
V_{c}^{2}=\pi^{2} \frac{K_{1}}{\varepsilon_{0} \varepsilon_{a}}\left[1+\frac{1}{4} \frac{K_{3}}{K_{1}}-\frac{1}{2} \frac{K_{2}}{K_{1}}\left(1+\frac{q d}{\pi / 2}\right)\right] .
$$

In Figure $15, V_{c}^{2}$ is plotted as a function of $q d$. From the slope of the linear fit, we deduced

$$
\frac{K_{2}}{\varepsilon_{0} \varepsilon_{a}}=0.068 \pm 0.002 \mathrm{~V}^{2} \text {. }
$$

Finally, we measured the spinodal voltage of the nematic phase in homeotropic samples of various thicknesses as a function of the temperature (or $q$ ). We recall that the nematic-to-cholesteric transition is usually first order (for a review, see $[2,12])$. The spinodal limit of the nematic phase can be calculated exactly and reads

$$
V_{0}^{2}=\frac{K_{2}}{\varepsilon_{0} \varepsilon_{a}} \frac{K_{2}}{K_{3}}(q d)^{2}-\pi^{2} \frac{K_{3}}{\varepsilon_{0} \varepsilon_{a}} .
$$

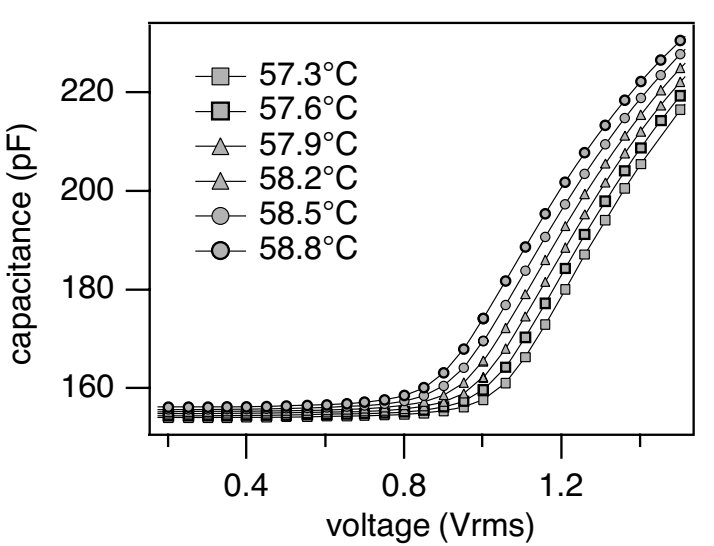

Fig. 14. Cell capacitance as a function of the applied voltage $(\pi / 2$-twisted sample, $d=6.8 \mu \mathrm{m}, f=10 \mathrm{kHz})$. The solid lines are just guides for the eyes.

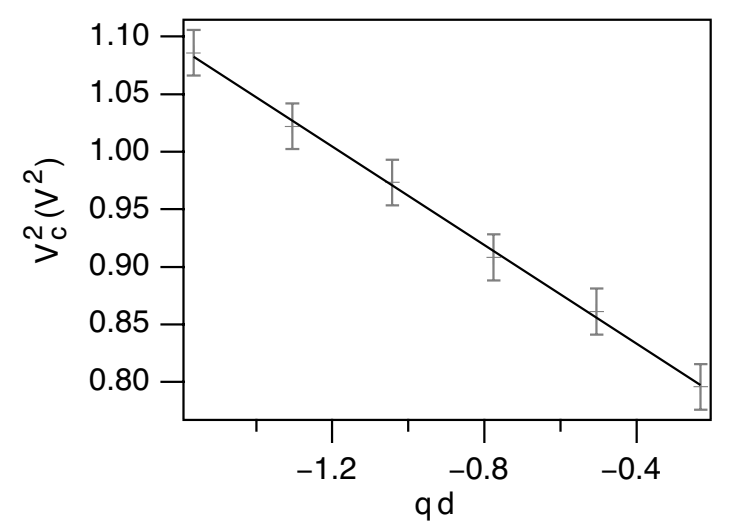

Fig. 15. Square of the critical voltage measured in a $\pi / 2$ twisted sample as a function of the equilibrium twist of the cholesteric. The solid line is the best fit to a linear law.

Below $V_{0}$, the nematic phase destabilizes and quickly gives a translationally invariant configuration (TIC) which then slowly modulates to form cholesteric fingers $(\mathrm{CF})$. Because $V_{0}$ is very sensitive to the sample thickness, a special care was taken during the preparation of the samples to limit their thickness variations (in practice, constant within $\pm 1 \mu \mathrm{m})$. We also worked in our home-made oven to control at best the temperature (within $0.01^{\circ} \mathrm{C}$ ). The experiment was performed with samples of different thicknesses, ranging between 10 and $75 \mu \mathrm{m}$. All our data are collected in Figure 16, in which we plotted $V_{0}^{2}$ as a function of $q d$. The best fit to equation (22) led to

$$
\frac{K_{3}}{\varepsilon_{0} \varepsilon_{a}}=0.15 \pm 0.02 \mathrm{~V}^{2}
$$

and

$$
\frac{K_{2}^{2}}{K_{3} \varepsilon_{0} \varepsilon_{a}}=0.0312 \pm 0.0006 \mathrm{~V}^{2} .
$$

It is worth noting that there is no data near the origin. This is normal inasmuch as the unwound (or "nematic") state is stable when the equilibrium pitch is typically larger than the sample thickness. Consequently, the 


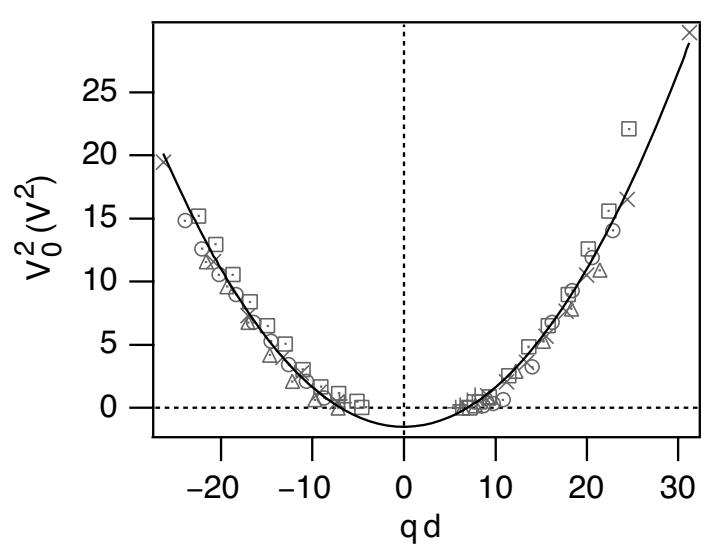

Fig. 16. Square of the spinodal voltage as a function of the product of the thickness and the equilibrium twist of the cholesteric. The sample thickness was chosen between 10 and $75 \mu \mathrm{m}$. The solid line is the best fit to equation (22).

previous value of $K_{3}$ given by the $Y$ value in the fit curve at $X=0$ is extrapolated.

Finally, note that we neglected in all this section the variations of the material parameters with respect to the temperature, which is justified within our experimental errors near the compensation temperature.

\section{Lehmann coefficient}

From the measurements described in the two previous sections, we can calculate all the materials constants, including the Lehmann coefficient $\nu$. Some of the measured quantities being coupled (for instance, we measured $K_{1}, K_{3}$ but also their ratio $\left.K_{3} / K_{1}\right)$, the error estimation is not simple and was determined by using the maximum-likelihood method [22]. This global method allowed us to calculate the most probable value and the $68 \%$ confidence interval for the elastic constants: $K_{1}=$ $(3.4 \pm 0.4) \times 10^{-12} \mathrm{~N}, K_{2}=(2.8 \pm 0.2) \times 10^{-12} \mathrm{~N}$ and $K_{3}=(5.9 \pm 0.6) \times 10^{-12} \mathrm{~N}$, and for the Lehmann coefficient [23]:

$$
\nu=(2.8 \pm 0.6) \times 10^{-7} \mathrm{~kg} \mathrm{~K}^{-1} \mathrm{~s}^{-2} .
$$

This last result is important as it clearly shows that the Lehmann coefficient does not vanish at the compensation temperature, in agreement with the previous results of Éber and Jánossy $[6,7]$.

Note, to end this section, that the only measurements in homeotropic anchoring would lead to $\nu=(2.9 \pm 0.6) \times$ $10^{-7} \mathrm{~kg} \mathrm{~K}^{-1} \mathrm{~s}^{-2}$ while those in planar anchoring (less precise) give $\nu=(2.2 \pm 0.9) \times 10^{-7} \mathrm{~kg} \mathrm{~K}^{-1} \mathrm{~s}^{-2}$. These two values are compatible within experimental errors, which reinforces our results.

To prove more convincingly that the Lehmann coefficient is different from 0 at the compensation temperature, we performed a new experiment using a planar gliding anchoring. This preliminary experiment is presented in the next section and reveals a continuous rotation of the director in a temperature gradient.

\section{Direct evidence of the Lehmann rotation: preliminary results}

As we mention in the introduction, the main problem to observe the continuous Lehmann rotation is to make samples in which the director is allowed to freely rotate at the boundaries. This requires to prepare gliding anchoring on the glass plates, which is a real experimental challenge. In this section, we describe our protocol to prepare and optically characterize such an anchoring. We then describe a preliminary experiment which clearly shows the Lehmann rotation at the compensation point. Finally, a model is presented, which reproduces quite well the experimental observations.

\subsection{Planar gliding anchoring: preparation and optical characterization}

Two surface treatments have already been described in the literature to obtain a planar gliding anchoring. "Gliding" means that the director is "free" to rotate in the azimuthal plane, without "solid friction". On the other hand, this does not exclude a viscous friction at the surface. The first gliding anchoring was found by Dozov et al. [24] and consists of treating the glass plates with a thin layer of (3glycidoxypropyl) trimethoxysilane (3-GPS). The second one, better according to Blanc et al. [25], consists of covering the glass with the commercial photopolymer NOA 60 (provided by Norland Optics) which is then polymerized by exposure to UV light. We thus bought this glue and tried to reproduce this surface treatment. For some unknown reasons, we did not succeed in preparing a good gliding anchoring in this way. We thus tried a new surface treatment which turned out to give good results.

Our protocol was the following. It consisted of spreading by spin-coating a thin layer of the hardener of an epoxy glue (structalit7 from Eleco). The latter was first dissolved in a ketone, the 2 -butanone ( $5 \%$ in mass of hardener). The solution was then filtered through $0.2 \mu \mathrm{m}$ PTFE membrane to eliminate dust particles and then spread by spincoating on the glass plate at $500 \mathrm{rpm}$ for $1 \mathrm{~min}$. At this concentration, the resin formed a homogeneous thin layer after evaporation of the ketone.

To characterize this surface treatment, we prepared a sample with the top glass plate treated in this way, whereas the bottom plate was treated in strong planar anchoring (rubbed polyimide). The sample thickness was $10 \mu \mathrm{m}$ and the mixture $8 \mathrm{OCB}+\mathrm{CC}$ was introduced by capillarity in the cholesteric phase. All measurements were performed in a Mettler oven. To rapidly eliminate the oily streaks which form after filling, the sample was rapidly heated into the isotropic phase and then cooled down into the cholesteric phase. Observations in white light under the microscope showed that the sample became rapidly homogeneous (in less than $1 \mathrm{~min}$ ), without any visible texture. On the other hand, its color between crossed polarizers was function of the temperature, indicating that the director was rotating on the top glass plate when the temperature was changed. 


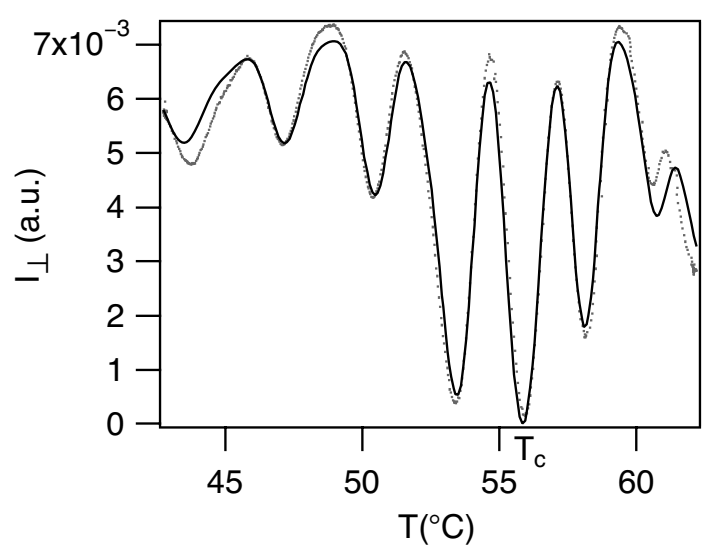

Fig. 17. Transmitted intensity between crossed polarizer and analyzer as a function of the temperature $(d=10 \mu \mathrm{m})$. Dots are the experimental points. The solid line is the best fit to equation (23).

To characterize this new surface treatment, we performed two experiments under the microscope.

First, we increased by successive increments of $0.2^{\circ} \mathrm{C}$ the temperature from the compensation temperature. At each temperature, we visually searched for the analyzer position for which the transmitted intensity was minimum when the polarizer was parallel to the anchoring direction on the bottom plate. Observations were made in green light $(\lambda=546 \mathrm{~nm})$. At the compensation temperature, we checked that the extinction was obtained between crossed polarizer and analyzer, which was characteristic of a nematic phase. By increasing the temperature, we observed that we needed to rotate the analyzer anticlockwise to get the minimum intensity, showing that the director was rotating in the same direction on the top glass plate (to a first approximation, the plane of polarization of the light follows the director rotation when the cholesteric pitch $p$ is larger than $d \Delta n$ ). In this way, its was possible to determine the sign of $q$ : positive above $T_{c}$ and negative below it.

The second experiment was more quantitative as we measured with a photodiode the transmitted intensity across the sample between crossed polarizer and analyzer as a function of the temperature. The polarizer was parallel to the anchoring direction at the bottom plate. A temperature ramp was imposed by a Mettler oven. The temperature was measured with a thermocouple placed beside the sample. The temperature and the light intensity were measured sequentially every $1 \mathrm{~s}$ with a Keithley 2001 multimeter equipped with a scanner card. Apart from systematic small temperature shifts (smaller than $0.5^{\circ} \mathrm{C}$ ) mainly due (in our opinion) to the fact that the thermocouple was not in contact with the liquid crystal, all curves we obtained were similar whatever the heating (or cooling) speed (chosen between $0.5^{\circ} \mathrm{C} / \mathrm{min}$ and $5^{\circ} \mathrm{C} / \mathrm{min}$ ). One typical curve is shown in Figure 17. It has an oscillating shape with an absolute minimum (corresponding to a complete extinction) at $T_{c}$. We shall note that in this example, the values of $T_{c}$ and of the clearing temperature were smaller than expected by about $3-4{ }^{\circ} \mathrm{C}$. This was certainly due to a problem of pollution of the liquid crystal when it comes in contact with the hardener of the epoxy glue. On the other hand, we observed that $T_{c}$ did not change significantly during many hours once the sample was prepared. In order to analyze this curve, we calculated the expression of the transmitted intensity by assuming a planar gliding anchoring at the top glass plate. We found

$$
\begin{aligned}
\frac{I}{I_{0}}= & \frac{1}{2}-\cos (2 q d) \frac{\kappa^{2}+\cos \left(2 q d \sqrt{1+\kappa^{2}}\right)}{2\left(1+\kappa^{2}\right)} \\
& -\sin (2 q d) \frac{\sin \left(2 q d \sqrt{1+\kappa^{2}}\right)}{2 \sqrt{1+\kappa^{2}}},
\end{aligned}
$$

with $\kappa=\frac{2 \pi}{\lambda} \frac{n_{e}-n_{o}}{2 q}=\frac{k \Delta n}{2 q}$. To fit our data, we chose as fit parameters the compensation temperature $T_{c}$ (assuming that the equilibrium twist is still given by $q\left(\mu \mathrm{m}^{-1}\right)=$ $0.1365\left(T-T_{c}\right)+0.0019\left(T-T_{c}\right)^{2}$, with the temperatures in ${ }^{\circ} \mathrm{C}$, see Sect. 4), the sample thickness $d$, the intensity $I_{0}$ and the birefringence we took in the form $\Delta n=a-b(T-$ $\left.T_{c}\right)$. The fit of the experimental curve to this law is good as seen in Figure 17. It led to $T_{c}=55,9^{\circ} \mathrm{C}, I_{0}=7,3 \times 10^{-3}$, $d=9.1 \mu \mathrm{m}$, and $\Delta n=0.083-0.0021\left(T-T_{c}\right)$. Note that the values of the thickness and of the birefringence at the compensation point found from the fit are close to the expected values: $9.1 \mu \mathrm{m}$ instead of $10 \mu \mathrm{m}$ for the thickness and 0.083 instead of 0.09 for the birefringence. These results made us confident in our initial assumption that the new anchoring is planar and gliding. On the other hand, we observed that it was degraded after one day (hysteresis and memory effects occurred). This is not surprising as the hardener of the epoxy glue (which is not polymerized) certainly slowly dissolves in the liquid crystal. We will not describe these aging effects in this paper because they are out of the scope of our study.

\subsection{Experimental evidence of a continuous Lehmann rotation at the compensation point}

In order to observe the Lehmann effect, we prepared a $10 \mu \mathrm{m}$-thick sample with the two glass plates treated for gliding planar anchoring. The sample was filled with the cholesteric mixture and annealed at the compensation temperature during one hour in a Mettler oven without the cover. Immediately after filling, the sample was full of disclination lines perpendicular to the glass plates, forming a typical structure à noyaux or "Schlieren texture" characteristic of the nematic phase. Due to their mutual attraction, the disclination lines of opposite signs progressively annihilated. After one hour, the sample was almost free of disclination lines with "large" zones (of a few tenths of $\mathrm{mm}$ in size) in which the director orientation was everywhere approximately the same. The next step was to impose a vertical temperature gradient while maintaining the sample at the compensation temperature. To do this, we started to blow air onto the top of the sample while increasing the temperature of the oven. To maintain the liquid crystal at the compensation temperature, we simultaneously observed its optical texture under the microscope. This method, although very simple, was efficient 

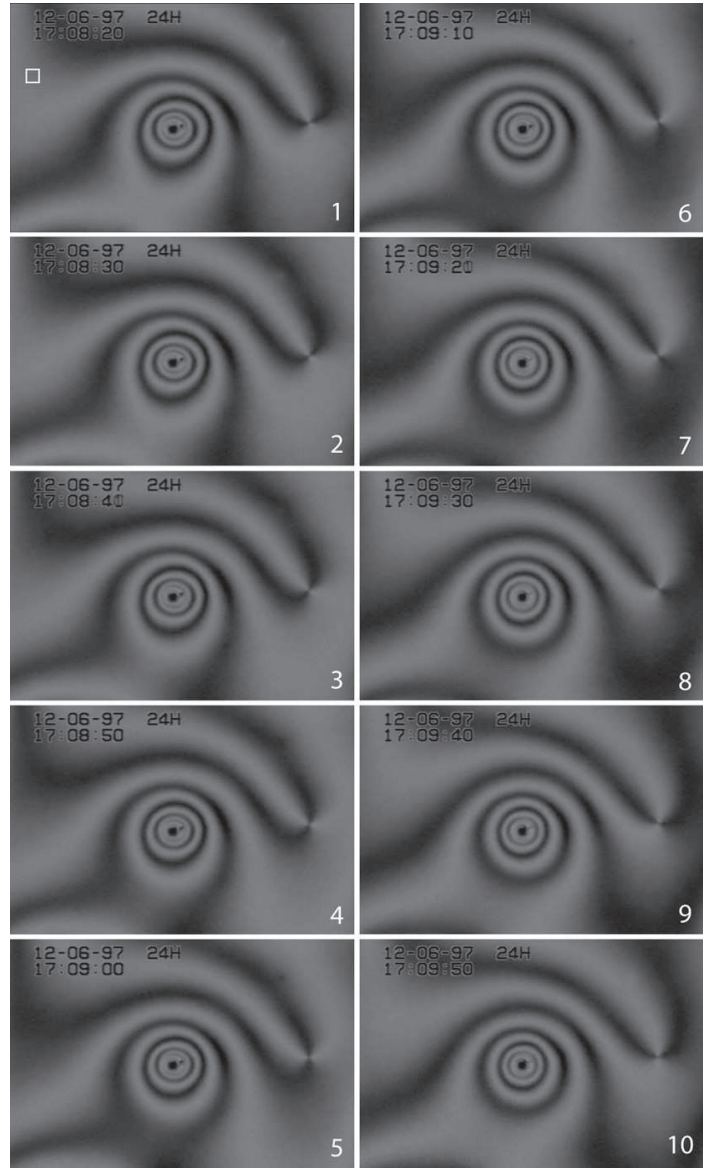

Fig. 18. Zone inside the sample where the director rotates continuously by forming concentric rings encircling a central defect. The rings propagate inward and collapse on the defect. The square in the first photograph marks the place where we measured the intensity (see Fig. 19). Close to the right side of the photos, one can see a disclination line, the two extinction branches of which rotate at constant angular velocity. Photos are taken between crossed polarizer and analyzer. Each photo is $450 \mu \mathrm{m}$ wide.

because the grey level of the extinction branches starting from the cores of the disclination lines (which are almost black at the compensation temperature) is very sensitive to the temperature. In this way, it was possible to increase the temperature of the oven by about $5^{\circ} \mathrm{C}$ above the compensation temperature, thus imposing a vertical temperature gradient to the liquid crystal layer. We then started to record with a video camera the time evolution of the texture. We immediately observed that the branches of the disclination lines were rotating, generating black and white fringes. This observation revealed that the director was continuously rotating under the action of the temperature gradient. At some places, the fringes formed rings encircling small defects of the surface treatment (certainly due to a dewetting of the hardener of the epoxy resin). At these places, the rings were moving continuously inward and collapsed in the centre as shown in Figure 18. To determine the periodicity of the process we measured the transmitted intensity at one point of the sample (more

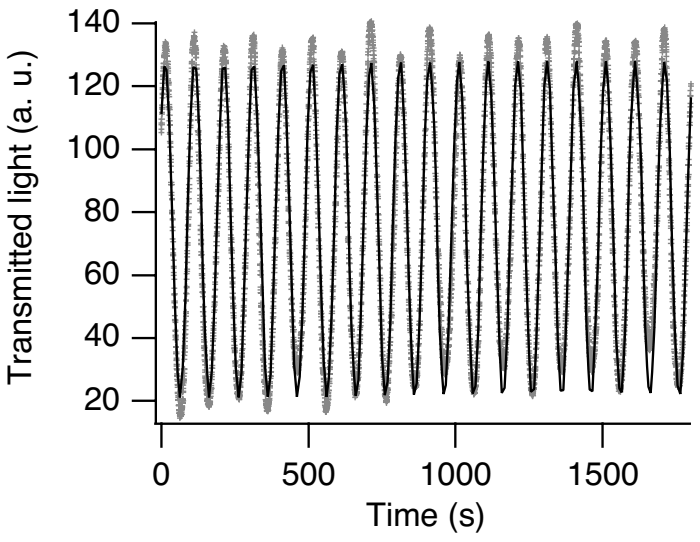

Fig. 19. Intensity measured with a camera video inside the square shown in Figure 18. Crosses are experimental points and the solid line is the best fit to a sinusoidal law of period $T=$ $100 \mathrm{~s}$. Only one-third of the recording is represented. During the full recording the director rotated locally by about $25 \pi$.

exactly inside a small square shown in Fig. 18) as a function of time (Fig. 19). This graph shows that the period, which corresponds to the passage of one fringe - and thus, to a rotation of the director by $\pi / 2$-is close to $T \approx 100 \mathrm{~s}$. We also checked qualitatively that the rotation was slowing down when the temperature gradient was decreased. On the other hand, it was impossible with our setup to reverse the temperature gradient to check that it was possible to unwind the director field. In the following subsection a very simple model is developed to explain this observation.

\subsection{Comparison with the theoretical model}

One supposes that the director lies in the $(x, y)$-plane and is invariant by translation along the $z$-axis. To simplify, we assume that $K_{1}=K_{3} \equiv K$ and that backflow effects are negligible. In this case, the torque equation becomes:

$$
\gamma_{1} \frac{\partial \varphi}{\partial t}=-\nu G+K\left(\frac{\partial^{2} \varphi}{\partial r^{2}}+\frac{1}{r} \frac{\partial \varphi}{\partial r}+\frac{1}{r^{2}} \frac{\partial^{2} \varphi}{\partial \theta^{2}}\right)
$$

where $\gamma_{1}$ is the local rotational viscosity, $\varphi$ the angle between the director and the $x$-axis, and $(r, \theta)$ the polar coordinates.

In order to model the central anchoring defect, we assume that its main effect is to locally increase the liquid crystal viscosity. Under this assumption, viscosity $\gamma_{1}$ in equation (24) becomes a function of $r$ of the type:

$$
\gamma_{1}+\delta \gamma_{1} e^{-\left(\frac{r}{r_{0}}\right)^{2}}
$$

where $r_{0}$ represents the typical radius of the defect. Solving with Mathematica equation (24) with $\gamma_{1}$ given by 
equation (24) yields

$$
\begin{aligned}
\varphi= & a-\frac{\nu G}{b} t \\
& -\nu G \frac{\delta \gamma_{1}}{b} \frac{r_{0}^{2}}{4 K}\left[C+\Gamma\left(0, \frac{r^{2}}{r_{0}^{2}}\right)-\log \left(\frac{r^{2}}{r_{0}^{2}}\right)+c \log (r)\right] \\
& -\nu G \frac{\gamma_{1}-b}{b} \frac{r^{2}}{4 K} .
\end{aligned}
$$

In this expression, $C$ is the Euler constant, $\Gamma$ the incomplete Euler function, and $a, b$, and $c$ are integration constants. In order that $\varphi$ does not diverge at the center of the defect, one must take $c=0$. Constant $a$ corresponds to a phase shift. It may be vanished by changing the time origin. In the following, we shall take $a=0$. Finally, one must take $b=\gamma_{1}$ to cancel the torque $\partial \varphi / \partial r$ at infinity. With this choice of the constants, the solution reads

$$
\varphi=-\frac{\nu G}{\gamma_{1}} t-\nu G \frac{\delta \gamma_{1}}{\gamma_{1}} \frac{r_{0}^{2}}{4 K}\left[C+\Gamma\left(0, \frac{r^{2}}{r_{0}^{2}}\right)+\log \left(\frac{r^{2}}{r_{0}^{2}}\right)\right] \text {. }
$$

This solution contains two terms. The first one is just a function of time: it gives the period $T$ of the process which is the time interval between the passage of two black fringes at the same point of the sample. As there is extinction when the director is either parallel or perpendicular to the polarizer, we obtain

$$
T=\frac{\pi}{2} \frac{\gamma_{1}}{\nu|G|}
$$

where we introduced $|G|$ as $G$ may be positive or negative (in our previous experiment, $G$ was negative as we heated from below). The second term depends on the spatial coordinate $r$ only. It gives the spatial organization of the director and the number of rings (between crossed polarizers) around the defects. We shall note that the larger $\delta \gamma_{1}$, the larger is the number of rings. On the other hand, period $T$ is independent of the "strength" of the central defect, which makes this quantity very interesting to measure experimentally as it only depends on the Lehmann coefficient $\nu$ and on the rotational viscosity $\gamma_{1}$.

This solution may be generalized in order to take into account the presence of a disclination line on the right of the central defect as observed experimentally in Figure 18. In this case, the solution is obtained by adding to solution (27) the quantity $m \theta_{d}$ where $m$ is the topological rank of the defect and $\theta_{d}$ the polar angle measured from the core of the disclination line. This solution is represented in Figure 20 over one period by taking $m=-1 / 2$ (with $\nu>0$ and $G<0$ ). We note that the simulation is very close to the experiment and that the sign of $m$ was chosen in order that the two extinction branches starting from the core of the line rotate in the same sense as in the experiment. Unfortunately, we cannot deduce from this preliminary experiment the value of the Lehmann coefficient because the temperature gradient in the liquid crystal layer is largely unknown. In addition, although we measured viscosity $\gamma_{1}$ in a previous article [20], its value may be different in the present sample because of the pollution by the glue. Thus, the next step will be to check

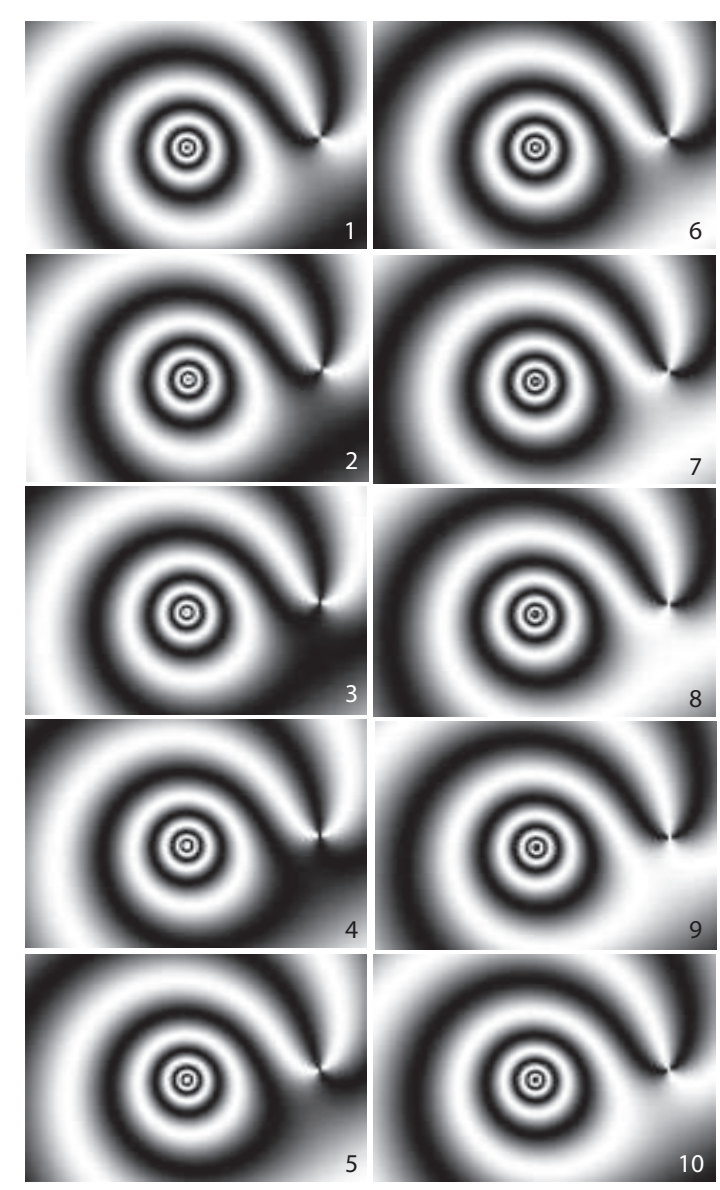

Fig. 20. Representation (between crossed polarizers) of the solution calculated numerically. The parameter $\delta \gamma_{1}$ has been chosen in order that the number of rings be approximately the same as in experiment.

the viscosity value and to make a special setup to impose a controlled temperature gradient.

\section{Conclusion}

In conclusion, we have found, in agreement with the pioneer work of Éber and Jánossy, that the Lehmann coefficient does not vanish at the compensation temperature in a cholesteric liquid crystal. We additionally found that the Lehmann coefficient is independent of the equilibrium twist $q$ within our experimental resolution. On the other hand, we cannot say anything about a possible dependence of this coefficient on the actual twist $\mathbf{n} \cdot \nabla \times \mathbf{n}$ as in our experiments with fixed boundary conditions the cholesteric phase is almost completely unwound because of the strong (homeotropic or planar) anchoring on the glass plates. These results experimentally prove the existence of a Lehmann effect of molecular origin which is certainly strongly reinforced by the collective behavior of the molecules responsible for the quadrupolar order of the phase. This conclusion is in disagreement with the theory defended by Pleiner and Brand. Indeed, these authors 
would be right if, as they claim in reference [9], the symmetries of a compensated cholesteric were exactly the same as those of a usual nematic phase. This is incorrect because a compensated cholesteric made of chiral molecules does not have symmetry planes and inversion centres. The same reasoning applies to an isotropic solution of chiral molecules and this is why such a solution has a rotatory power contrary to isotropic liquids made of achiral molecules.

We would also like to emphasize that we determined this coefficient in two different geometries (homeotropic, but also planar, which is new) and that our measurements of the material constants were often redundant, but always compatible with one another. In addition, the two experiments yielded similar results as we have emphasized before.

A well-informed reader could also object that our results contradict those of Madhusudana et al. [26,27] about the electric Lehmann effect at the compensation point. For this reason, we redid their experiment with our mixture. Although our observations were essentially the same as theirs, we showed that they could not result from an electric Lehmann effect, but more simply from flexoelectricity [20].

Finally, we observed a continuous Lehmann rotation of the director at the compensation temperature in a sample treated on both sides for planar gliding anchoring. The next step is now to make a new experimental setup to study the Lehmann rotation in samples treated with this new surface treatment. One crucial point will be to measure accurately the vertical temperature gradient, for instance by using contact thermocouples. The setup will also have to allow the possibility of reversing the temperature gradient. Measurements of the thermal conductivity of the liquid crystal will also be necessary to calculate the exact temperature gradient inside the liquid crystal. Finally, we plan to improve and to better characterize the surface treatment which strongly pollutes the liquid crystal for the moment.

We thank Yves Pomeau, Sriram Ramaswamy and Pawel Pieranski for helpful discussions. This work has been supported by the Polonium Program No. 11622QC.

\section{References}

1. P.-G. de Gennes, J. Prost, The Physics of Liquid Crystals (Oxford University Press, Oxford, 1995).

2. P. Oswald, P. Pieranski, Nematic and Cholesteric Liquid Crystals: Concepts and Physical Properties Illustrated by Experiments (Taylor \& Francis, CRC press, Boca Raton, 2005).

3. O. Lehmann, Ann. Phys. (Leipzig) 2, 649 (1900).
4. O. Lehmann, Flüssige Kristalle und ihr Scheinbares Leben (Verlag von Leopold Voss, Leipzig, 1921).

5. F.M. Leslie, Proc. R. Soc. London, Ser. A 307, 359 (1968).

6. N. Éber, I. Jánossy, Mol. Cryst. Liq. Cryst. Lett. 72, 233 (1982).

7. N. Éber, I. Jánossy, Mol. Cryst. Liq. Cryst. Lett. 102, 311 (1984).

8. H. Pleiner, H.R. Brand, Mol. Cryst. Liq. Cryst. Lett. 5, 61 (1987).

9. H. Pleiner, H.R. Brand, Mol. Cryst. Liq. Cryst. Lett. 5, 183 (1988).

10. N. Éber, I. Jánossy, Mol. Cryst. Liq. Cryst. Lett. 5, 81 (1988).

11. A. Dequidt, P. Oswald, Europhys. Lett. 80, 26001 (2007).

12. P. Oswald, J. Baudry, S. Pirkl, Phys. Rep. 337, 67 (2000).

13. F.J. Kahn, Appl. Phys. Lett. 22, 386 (1973).

14. P. Oswald, P.M. Moulin, P. Metz, J.-C. Géminard, P. Sotta, J. Phys. III 3, 1891 (1993).

15. K.C. Lim, J.T. Ho, Mol. Cryst. Liq. Cryst. 47, 173 (1978).

16. As pointed out by Pawel Pieranski, convection must develop in the sample as long as it is submitted to a horizontal temperature gradient. As a result, the director field must distort in homeotropic samples because of the shear flow. It can be checked that this effect should lead to a phase shift $\Phi_{\mathrm{H}}$ proportional to $d^{9}$. This dependence is very different from that observed experimentally $\left(\Phi_{\mathrm{H}} \propto d^{5}\right)$, suggesting that hydrodynamic effects are negligible in our experiments. This conclusion agrees with the experimental results of Éber and Jánossy [6] who found that $\Phi_{\mathrm{H}}=0$ at all temperature gradients in a pure nematic $(8 \mathrm{CB})$.

17. We emphasize that in the planar geometry the convection does not destabilize the director field as the director is perpendicular to the shear plane.

18. S.W. Morris, P. Palffy-Muhoray, D.A. Balzarini, Mol. Cryst. Liq. Cryst. 139, 263 (1986).

19. C.V. Brown, N.J. Mottram, Phys. Rev. E 68, 031702 (2003).

20. A. Dequidt, P. Oswald, Eur. Phys. J. E 24, 157 (2007).

21. E.P. Raynes, Mol. Cryst. Liq. Cryst. Lett. 4, 1 (1986).

22. D. Taupin, Probabilities Data Reduction and Error Analysis in the Physical Sciences (Les Editions de Physique, Les Ulis, 1988).

23. Note that we have implicitly assumed that $\nu_{e f f}$ is positive as in the mixture $8 \mathrm{CB}+\mathrm{CC}$, for which this result was proved experimentally by Éber and Jánossy in reference [7]. We emphasize that taking $\nu_{\text {eff }}<0$ would have lead to $\nu=-10.4 \times 10^{-7} \mathrm{~kg} \mathrm{~K}^{-1} \mathrm{~s}^{-2}$, which is more than three times larger in absolute value than the retained value. The conclusion is that, in all cases, $\nu$ is different from 0 , which is the main result of this article.

24. I. Dozov, D.N. Stoenescu, S. Lamarque-Forget, Ph. Martinot-Lagarde, E. Polossat, Appl. Phys. Lett. 77, 4124 (2000).

25. C. Blanc, D. Svenšek, S. Žumer, M. Nobili, Phys. Rev. Lett. 95, 097802 (2005).

26. N.V. Madhusudana, R. Pratibha, H.P. Padmini, Mol. Cryst. Liq. Cryst. 202, 35 (1991).

27. H.P. Padmini, N.V. Madhusudana, Liq. Cryst. 14, 497 (1993). 